\title{
Standard Modifiable Cardiovascular Risk Factors and Prognosis of Acute Coronary Syndrome in Younger Patients
}

\author{
Xu Chen, Han-xiong Liu, Xiu-qiong Yu, Si-qi Yang, Ling-yao Qi and Lin Cai \\ Department of Cardiology, The Third People's Hospital of Chengdu, Affiliated Hospital of Southwest Jiaotong University, Chengdu, \\ Sichuan, China
}

\begin{abstract}
Objective: To investigate standard modifiable cardiovascular risk factors (SMuRFs) and prognosis of patients with acute coronary syndrome (ACS) aged 50 years or younger.

Study Design: An observational study.

Place and Duration of Study: Eleven general hospitals in Chengdu, Sichuan Province, China, from January 2017 to June 2019. Methodology: Patients with ACS were stratified into younger group ( $\leq 50$ years) and older group ( $>50$ years). The baseline characteristics and prognosis were compared for two groups. Survival analysis was used to assess the long-term prognosis.

Results: Among a total of 1982 ACS patients, $322(16.2 \%)$ were of $\leq 50$ years. Compared with older patients, younger patients were more likely to have at least one SMuRFs $(90.0 \%$ vs. $84.3 \%, p=0.013)$. The younger group had a higher prevalence of smoking $(62.8 \%$ vs. $34.1 \%, p<0.001)$ and hypercholesterolemia $(36.2 \%$ vs. $23.4 \%, p<0.001)$ compared with the older group. Younger male patients were more likely to have at least one SMuRFs than younger female patients $(91.6 \%$ vs. $74.1 \%, p=$ $0.011)$. After the follow-up of $15(10,22)$ months, the cumulative rates of major adverse cardiovascular and cerebrovascular events (MACCE) of the younger patients were significantly lower than those in the older patients [hazard ratio (HR): 0.2661 , 95\% confidence interval (Cl): $0.1932-0.3665, p<0.001]$.

Conclusion: Younger patients with ACS were more likely to have at least one SMuRFs; and were likely to have a better prognosis than older patients.
\end{abstract}

Key Words: Coronary artery disease, Acute coronary syndrome, Middle aged, Risk factors, Prognosis.

How to cite this article: Chen X, Liu HX, Yu XQ, Yang SQ, Qi LY, Cai L. Standard Modifiable Cardiovascular Risk Factors and Prognosis of Acute Coronary Syndrome in Younger Patients. J Coll Physicians Surg Pak 2021; 31(12):1394-1398.

\section{INTRODUCTION}

The incidence and mortality of coronary heart disease (CHD) in the elderly population have recently tended to decrease in most countries. However, a similar decline has not been observed among young individuals. ${ }^{1}$ The incidence of acute myocardial infarction (AMI) in young population in China has been shown an increase, as reported by a recent study. ${ }^{2}$ In addition, compared with young male patients with acute coronary syndrome (ACS), the hospitalisation rates among young female patients showed a more obvious upward trend. ${ }^{3}$

Correspondence to: Lin Cai, Department of Cardiology,

The Third People's Hospital of Chengdu, Affiliated

Hospital of Southwest Jiaotong University, Chengdu,

Sichuan, China

E-mail: clin63@hotmail.com

Received: September 02, 2021; Revised: November 11, 2021;

Accepted: November 11, 2021

DOI: https://doi.org/10.29271/jcpsp.2021.12.1394
The standard modifiable cardiovascular risk factors (SMURFs), including hypertension, diabetes mellitus, hypercholesterolaemia, and smoking are vital elements of the Framingham cardiovascular risk score and were identified in epidemiological studies in the 1960s. ${ }^{4,5}$ Studies have shown that in some middleand high-income countries, the application of controlling modifiable cardiovascular risk factors in primary/secondary prevention has resulted in significant reductions in the morbidity and mortality of $\mathrm{CHD} .{ }^{6}$ However, only few studies have been performed on the burden of SMuRFs in young ACS patients. By providing evidence for the primary and secondary prevention strategiesforyoung individuals could help further reduce the incidence and mortality of ACS in the young population.

Therefore, the aim of this study was to investigate the SMuRFs profile, diagnosis, treatment status, and prognosis of patients with ACS aged less than 50 years and further subdivided according to gender.

\section{METHODOLOGY}

It was a multi-centre observational study. The authors observed consecutive patients that were admitted to 11 general hospitals in China (namely The Third People's Hospital of Chengdu; The First 
People's Hospital of Chengdu; The Second People's Hospital of Chengdu; The People's Hospital of Dujiangyan; The People's Hospital of Pidu District; The First People's Hospital of Shuangliu District; The People's Hospital of Dayi County; The First People's Hospital of Jintang; The People's Hospital of Pengzhou; The First Affiliated Hospital of Chengdu Medical College; The People's Hospital of Chongzhou) from January 2017 to June 2019 with a diagnosis of presumptive acute coronary syndrome (ACS). ACS was defined according to guidelines issued by the Chinese Society of Cardiology for the diagnosis of patients with ST Elevation MI (STEMI), non-ST Elevation MI (NSTEMI) or unstable angina (UA). ${ }^{7.8}$ Theexclusion criteria were severe valvularheartdisease, constrictive pericarditis, myocarditis, non-ischemic cardiomyopathy, congenital heart disease, rheumatic heart disease or other serious heart diseases, malignant tumor; renal insufficiency, liver dysfunction, coronary vasculitis, systemic sclerosis, systemic vasculitis, systemic lupus erythematosus or other connective tissue diseases, severe Infection; respiratory failure or acute pulmonary embolism, missing important clinical trial data, and lost to follow-up. The patients were divided into two groups as younger patients ( $\leq 50$ years) and older patients ( $>50$ years).

Data were retrieved from the electronic health records (EHR) of each hospital. The collected data included demographic data, cardiac risk factors, past medical history, diagnoses, symptoms and clinical signs at admission, laboratory data on admission and angiographic data, type of revascularisation, and medication at hospital discharge. After the patients were discharged from the hospital, the in-hospital mortality of the study population was obtained by inquiring the discharge status of the patients through theEHRsystem.

A follow-up of the patients was performed at 1, 6 and 12 months after discharge; and annually thereafter, to obtain their major adverse cardiovascular and cerebrovascular events (MACCE) via EHRs ortelephone questionnaires.

The endpoints were the in-hospital mortality and MACCE during follow-up.

Current smoking was defined as smoking within six months prior to admission. Diabetes was defined as being previously diagnosed with diabetes mellitus or treated with a glucose-lowering agent. Hypertension was defined as being previously diagnosed with hypertension or treated with an antihypertensive agent. Hypercholesterolemia was defined as previous diagnosis of hypercholesterolemia, treatment with anti-hyperlipidemic agent, low-density lipoprotein cholesterol (LDL-C) $\geq 3.4 \mathrm{mmol} / \mathrm{L}$ at admission or total cholesterol (TC) $\geq 5.2 \mathrm{mmol} / \mathrm{L}$ at admission. MACCE included all-cause death, defined as cardiogenic and non-cardiogenic, myocardial infarction (MI), defined as a new myocardial infarction, coronary revascularization, defined as any percutaneous revascularization or surgical bypass of an epicardial vessel, and stroke, which was defined as a new cerebral infarction or hemorrhage diagnosed by a neurologist.

Categorical variables were expressed as counts and percentages, and the Chi-square or Fisher's Exact test were used to perform group comparisons. Kolmogorov Smirnov test was used for evaluation of the normality of continuous variables. Variables with normal distribution were expressed as mean \pm SD and group comparisons were performed using Student t-test. Variables with non-normal distribution were expressed as median (IQR) and analysed by using Mann-Whitney U-test. The Kaplan-Meier method was used to calculate cumulative event rates, which were compared between the groups using the log-rank test. All the statistical tests were two-tailed, and a $p$-value $<0.05$ was considered to be statistically significant. The data analysis was performed using the SPSS version 26.0 statistical software, the Kaplan-Meier survival curve was plotted using the GraphPad Prism 8software.

Table I: Baseline characteristics of the study population.

\begin{tabular}{|c|c|c|c|}
\hline Variable & $\begin{array}{c}\text { Younger patients } \\
(n=322)\end{array}$ & $\begin{array}{c}\text { Older patients } \\
(n=1660)\end{array}$ & p-value \\
\hline Age (years) & $46(41,48)$ & $70(62,78)$ & $<0.001$ \\
\hline Male & $289(89.8 \%)$ & $1139(68.6 \%)$ & $<0.001$ \\
\hline Previous stroke & $1(0.3 \%)$ & $92(5.6 \%)$ & $<0.001$ \\
\hline Previous smoking & $9(2.8 \%)$ & $195(11.8 \%)$ & $<0.001$ \\
\hline \multicolumn{4}{|l|}{ SMuRFs } \\
\hline Current smoking & $201(62.8 \%)$ & $564(34.1 \%)$ & $<0.001$ \\
\hline Hypertension & $125(38.8 \%)$ & $987(59.5 \%)$ & $<0.001$ \\
\hline Diabetes & $55(17.1 \%)$ & $459(27.7 \%)$ & $<0.001$ \\
\hline Hypercholesterolemia & $105(36.2 \%)$ & $354(23.4 \%)$ & $<0.001$ \\
\hline \multicolumn{4}{|l|}{ Number of SMuRFs } \\
\hline$\geq 1$ & $260(90.0 \%)$ & $1272(84.3 \%)$ & 0.013 \\
\hline$\geq 2$ & $137(47.4 \%)$ & $709(47.0 \%)$ & 0.896 \\
\hline$\geq 3$ & $39(13.5 \%)$ & $170(11.3 \%)$ & 0.279 \\
\hline 4 & $9(3.1 \%)$ & $11(0.7 \%)$ & $<0.001$ \\
\hline \multicolumn{4}{|l|}{ Clinical signs } \\
\hline Chest pain & $307(97.5 \%)$ & $1482(92.7 \%)$ & 0.002 \\
\hline $\begin{array}{l}\text { Systolic blood pressure } \\
(\mathrm{mmHg})\end{array}$ & $130(117,146)$ & $130(115,150)$ & 0.977 \\
\hline Heart rate (beats per min) & $78(70,93)$ & $76(66,89)$ & 0.003 \\
\hline STEMI & $194(60.2 \%)$ & $865(52.1 \%)$ & 0.007 \\
\hline NSTEMI & $57(17.7 \%)$ & $417(25.1 \%)$ & 0.004 \\
\hline UA & $71(22.0 \%)$ & $378(22.8 \%)$ & 0.777 \\
\hline \multicolumn{4}{|l|}{ Laboratory variables } \\
\hline Creatinine $(\mu \mathrm{mol} / \mathrm{L})$ & $\begin{array}{c}72.10 \\
(63.10,85.95) \\
\end{array}$ & $80.80(67.00,98.83)$ & $<0.001$ \\
\hline Triglycerides (mmol/L) & $1.92(1.19,2.99)$ & $1.33(0.94,1.96)$ & $<0.001$ \\
\hline Total cholesterol (mmol/L) & $4.74(3.95,5.40)$ & $4.24(3.57,5.00)$ & $<0.001$ \\
\hline $\mathrm{HDL}-\mathrm{C}(\mathrm{mmol} / \mathrm{L})$ & $1.06(0.86,1.33)$ & $1.13(0.95,1.36)$ & 0.005 \\
\hline LDL-C (mmol/L) & $2.98(2.31,3.51)$ & $2.54(2.00,3.18)$ & $<0.001$ \\
\hline AST (IU/L) & $\begin{array}{c}57.00 \\
(27.00,133.50) \\
\end{array}$ & $\begin{array}{c}44.45 \\
(25.00,128.18) \\
\end{array}$ & 0.136 \\
\hline $\operatorname{ALT}(I U / L)$ & $\begin{array}{c}38.00 \\
(23.00,60.05) \\
\end{array}$ & $28.00(18.70,45.33)$ & $<0.001$ \\
\hline Uric acid (umol/L) & $\begin{array}{c}402.90 \\
(333.00,479.50)\end{array}$ & $\begin{array}{c}369.00 \\
(300.00,447.00)\end{array}$ & $<0.001$ \\
\hline \multicolumn{4}{|l|}{ Diagnosis and treatment } \\
\hline Coronary angiography & $244(75.8 \%)$ & $1199(72.2 \%)$ & 0.190 \\
\hline $\begin{array}{l}\text { Mutiple coronary arteries } \\
\text { lesion }\end{array}$ & $52(28.9 \%)$ & $338(39.1 \%)$ & 0.010 \\
\hline Primary $\mathrm{PCl}$ & $168(52.5 \%)$ & $721(44.0 \%)$ & 0.005 \\
\hline Elective $\mathrm{PCl}$ & $32(10.0 \%)$ & $239(14.6 \%)$ & 0.030 \\
\hline Thrombolytic therapy & $2(0.6 \%)$ & $12(0.7 \%)$ & 0.835 \\
\hline Non-reperfusion therapy & $118(36.9 \%)$ & $667(40.7 \%)$ & 0.202 \\
\hline \multicolumn{4}{|c|}{ Medication at hospital discharge } \\
\hline Dual antiplatelet inhibitors & $237(90.8 \%)$ & $1179(90.1 \%)$ & 0.740 \\
\hline Statins & $267(95.0 \%)$ & $1370(95.8 \%)$ & 0.554 \\
\hline ACEI/ARB & $152(54.9 \%)$ & $802(56.6 \%)$ & 0.605 \\
\hline Beta blockers & $205(73.7 \%)$ & $975(68.3 \%)$ & 0.074 \\
\hline Acid suppression therapy & $87(31.1 \%)$ & $508(35.9 \%)$ & 0.124 \\
\hline \multicolumn{4}{|c|}{$\begin{array}{l}\text { SMURFS=standard modifiable cardiovascular risk factors, STEMI=ST segment elevation } \\
\text { myocardial infarction, NSTEMI=non-ST-segment elevation myocardial infarction, UA=unstable } \\
\text { angina, } H D L-C=\text { high-density lipoprotein cholesterol, } L D L-C=l o w \text {-density lipoprotein } \\
\text { cholesterol, } A S T=\text { aspartate aminotransferase, } A L T=\text { alanine aminotransferase, } \\
\text { ACEI=angiotensin-converting enzyme inhibitors, } A R B=\text { angiotensin receptor blockers. }\end{array}$} \\
\hline
\end{tabular}

RESULTS 
A total of 1,982, ACS patients were included in the study, among which 322 (16.2\%) were younger patients. The median age of all the patients was $67(55,76)$ years, most patients were males $(72.0 \%)$ and $53.4 \%$ of the patients were diagnosed with STEMI.

The baseline characteristics, diagnosis and treatment of the study population are listed in Table I. Compared with older patients, younger patients were more often males, more likely to smoke, more likely to be treated with primary $\mathrm{PCl}(p=0.005)$ and had higher levels of triglycerides (TG) $(p<0.001)$, TC $(p<0.001)$, LDL-C $(p<0.001)$ and uric acid $(p<0.001)$. In addition, younger patients had lower levels of high-density lipoprotein cholesterol $(H D L-C)(p=0.005)$ and were less likely to have multi-vessel coronary disease $(p=0.010)$.

Among the younger patients, $90.0 \%$ had at least one SMuRFs, which was a little higher than older patients $(p=0.013)$. Current smoking (62.8\%), hypertension (38.8\%) and hypercholesterolemia (36.2\%) were the most prevalent SMuRFs among younger patients. On the other hand, the dominant SMuRFs in older patients were hypertension (59.5\%) and current smoking (34.1\%), as listed in Table I

Table II: Gender differences in standard modifiable cardiovascular risk factors of the younger patients with ACS.

\begin{tabular}{|c|c|c|c|}
\hline Variable & Male $(n=289)$ & Female $(n=33)$ & p-value \\
\hline \multicolumn{4}{|l|}{ SMuRFs } \\
\hline Current smoking & $199(69.3 \%)$ & $2(6.1 \%)$ & $<0.001$ \\
\hline Hypertension & $108(37.4 \%)$ & $17(51.5 \%)$ & 0.114 \\
\hline Diabetes & 46 (15.9\%) & 9 (27.3\%) & 0.101 \\
\hline Hypercholesterolemia & $97(36.9 \%)$ & $8(29.6 \%)$ & 0.455 \\
\hline \multicolumn{4}{|l|}{ Number of SMuRFs } \\
\hline$\geq 1$ & $240(91.6 \%)$ & $20(74.1 \%)$ & 0.011 \\
\hline$\geq 2$ & $126(48.1 \%)$ & $11(40.7 \%)$ & 0.466 \\
\hline$\geq 3$ & $38(14.5 \%)$ & $1(3.7 \%)$ & 0.146 \\
\hline 4 & $9(3.4 \%)$ & $0(0.0 \%)$ & 1.000 \\
\hline
\end{tabular}

Table III: Short-term and long-term clinical events of the study population.

\begin{tabular}{|l|c|c|c|}
\hline Outcome & $\begin{array}{c}\text { Younger patients } \\
(\mathbf{n = 3 2 2})\end{array}$ & $\begin{array}{c}\text { Older patients } \\
(\mathbf{n}=\mathbf{1 6 6 0 )}\end{array}$ & $\mathbf{p}$-value \\
\hline in-hospital mortality & $7(2.2 \%)$ & $144(8.7 \%)$ & $<0.001$ \\
\hline $\begin{array}{l}\text { Events occurred } \\
\text { during follow-up }\end{array}$ & $\begin{array}{c}\text { Younger patients } \\
(\mathbf{n}=\mathbf{3 1 5})\end{array}$ & $\begin{array}{c}\text { Older patients } \\
(\mathbf{n}=\mathbf{1 5 1 6 )}\end{array}$ & \\
\hline MACCE & $14(4.4 \%)$ & $245(16.2 \%)$ & $<0.001$ \\
\hline All-cause death & $1(0.3 \%)$ & $135(8.9 \%)$ & $<0.001$ \\
\hline Myocardial infarction & $1(0.3 \%)$ & $10(0.7 \%)$ & 0.475 \\
\hline Coronary revascularization & $8(2.5 \%)$ & $67(4.4 \%)$ & 0.126 \\
\hline Stroke & $5(1.6 \%)$ & $39(2.6 \%)$ & 0.299 \\
\hline MACCE=major adverse cardiovascular and cerebrovascular events. \\
\hline
\end{tabular}

The subgroup analysis showed that in younger patients, more male patients had at least one SMuRFs than women $(91.6 \%$ vs. $74.1 \%, p=0.011)$. SMuRFs of younger male patients were still mainly current smoking (69.3\%), hypertension (37.4\%), and hypercholesterolemia (36.9\%), while hypertension $(51.5 \%)$ and hypercholesterolemia (29.6\%) were the most prevalent SMuRFs in younger women patients (Table II).

Among the 1,982 patients included in this study, the in-hospital mortality rate was $7.6 \%$. Specifically, the in-hospital mortality rate in younger patients was significantly lower than that in older patients $(2.2 \%$ vs. $8.7 \%, p<0.001)$, Table III. The remaining patients were followed up for a median of $15(10,22)$ months. During the follow-up, the cumulative incidence of MACCE in young group was $4.4 \%$. The cumulative incidence of MACCE in the two groups during follow-up was shown in the Kaplan-Meier curve (Figure 1). The cumulative incidence of MACCE was lower in younger patients compared with older patients [hazard ratio (HR): $0.2661,95 \%$ confidence interval (Cl): $0.1932-0.3665$, $\mathrm{p}<0.001]$. However, in the subgroup analysis, no gender difference in the prognosis was observed in theyounger group.

The cumulative incidence of MACCE was lower in younger patients compared with older patients [hazard ratio (HR): $0.2661,95 \%$ confidence interval $(\mathrm{Cl}): 0.1932-0.3665, \mathrm{p}<0.001$ ].

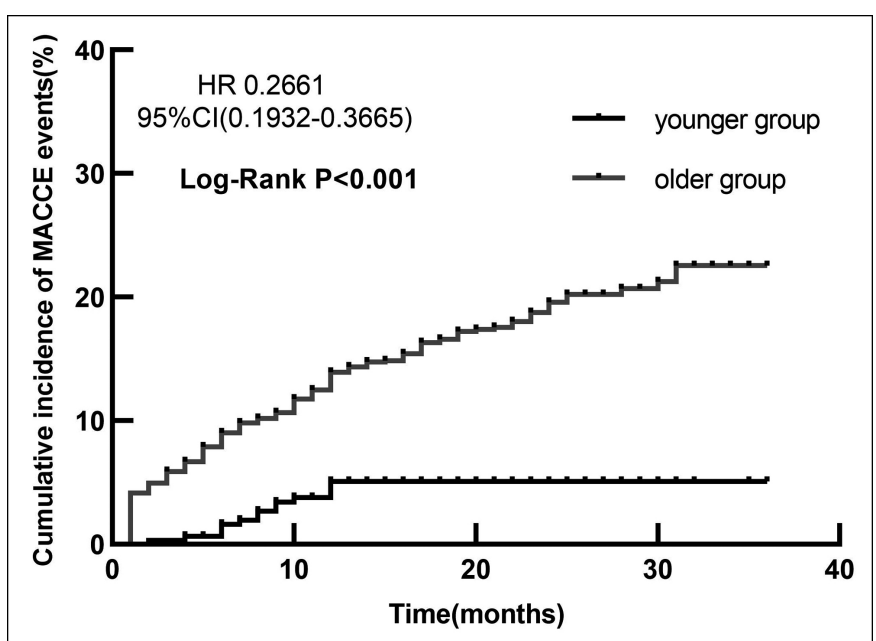

Figure 1: The cumulative incidence of MACCE in the younger group and oldergroup.

MACCE = Major adverse cardiovascular and cerebrovascular events.

\section{DISCUSSION}

In this study, it was found that the proportion of male patients and those receiving primary $\mathrm{PCl}$, was higher in younger ACS patients compared with older patients. Younger patients had a higher burden of SMuRFs, which were predominated by easily controlled factors, such as smoking and hypercholesterolemia. The prognosis of younger ACS patients seems better than that of older patients. However, it is still not good.

In consistence with previously reported results, ${ }^{9,10}$ this study found a higher proportion of male patients in the group of younger patients than that in the older patients' group. In the younger patients' group, male ACS patients accounted for $89.8 \%$, much higher than female patients. This large difference may be due to many reasons. Men have a higher burden of smoking exposure than women. According to serial cross-sectional National Health Service Surveys in China, smoking prevalence in men was $47.2 \%$ (46.9-47.5) in 2013, much higher than the $2.7 \%(2.6-2.8)$ in women. ${ }^{11}$ Another study has shown that the onset of ACS in smokers is earlier than that in non-smokers. ${ }^{12}$ Men have higher levels of exposure to dyslipidemia compared with women. Studies have shown that in young people, premenopausal women have lower blood lipid levels than men of the same age. ${ }^{13,14}$

Different from previous studies, ${ }^{15}$ this study found that the proportion of younger patients receiving primary $\mathrm{PCl}$ was higher 
than that of older patients. This may be due to the high prevalence of STEMI in the younger group compared with the older group. Studies have found that the proportion of STEMI patients receiving $\mathrm{PCl}$ was much higher than that of NSTEMI patients. ${ }^{16}$

Similar to the results of previous studies, ${ }^{15,17}$ the results of this study showed that $90.0 \%$ of the younger patients had at least one of the SMuRFs. Due to a higher prevalence of current smoking and hypercholesterolemia in younger patients with ACS, younger patients showed a higher burden of SMuRF$\mathrm{s}$ than older patients. This can be explained for two reasons. First, some older patients may have quitted smoking or started taking cholesterol-lowering drugs because they have a higher incidence of pre-existing coronary heart disease, hypertension and other complications. Another reason may be that greater social pressure leads to higher odds of smoking and poor dietary habitsamong younger patients.

The burden of modifiable risk factors in young patients with first AMI has been recently shown to increase, and modifiable risk factors were shown to be associated with an increased risk of recurrent $\mathrm{AMI}$ or fatal coronary heart disease events within 5 years. ${ }^{17-19}$ Therefore, the control of modifiable risk factors should be carried out in young ACS susceptible population to achieve primary prevention. In addition, secondary prevention strategies targeting modifiable risk factors should be applied to reduce the recurrence rate and mortality of coronary heart disease in high-risk patients in the younger population with existing diseases.

More than two-thirds of the younger male patients in the study were active smokers, while hypertension and diabetes were more common in younger female patients. In general, younger male patients had a heavier burden of SMuRFs than female patients. Compared with other SMuRFs, current smoking was the most easily controlled modifiable risk factor; quitting smoking reduced the individual's mortality risk in patients with CHD by about 36\%. ${ }^{20}$ The EUROASPIRE IV survey reported that about $90 \%$ of CHD patients in 24 European countries received smoking cessation interventions. ${ }^{21}$ Another survey showed that in recent years, the proportion of ACS patients receiving smoking cessation intervention in the United States has been maintained at nearly $100 \% .{ }^{22}$ However, the Improving Care for Cardiovascular Disease in China-Acute Coronary Syndrome Project reported that only $35.3 \%$ of ACS patients received smoking cessation intervention before being discharged, ${ }^{12}$ which is much lower than that of CHD patients in other countries. Therefore, for younger male patients with ACS, more attention should be paid to control smoking in secondary prevention.

Both short- and long-term outcomes in the younger group were better than those in the older group. However, the in-hospital mortality in young patients was still $2.2 \%$, and the cumulative MACCE rate during follow-up was $4.4 \%$. It was reported by a national cohort study in Norway that the incidence of AMI was lower in younger people, however, during 2.4 years of follow-up, of every $10 \mathrm{AMI}$ patients younger than 45 years, one died or experienced a new cardiovascular event. ${ }^{15}$ A study in China showed that younger patients with AMI had a higher incidence of bleeding events within 5 years compared with older patients. ${ }^{23}$ In people younger than 50 years old, ACS has a great impact on the patients' mental health and work ability and may impose a sizeable burden on family and society. Therefore, the prevention and treatment measures should be strengthened to further improve the quality of life and prognosis of younger patients with ACS.

Firstly, the sample size of younger ACS patients was small, and the study results may be biased. Secondly, the small number of young women makes it impossible to analyse gender differences in the clinical outcomes among young patients. Finally, the rate of loss to follow-up (LTFU) is high, so the follow-up results may be biased.

\section{CONCLUSION}

Compared with the older patients, younger patients with ACS were more likely to have at least one SMuRFs. Younger patients presented with a better short-term and long-term prognosis than older patients, but the prognosis was still poor. Therefore, controlling of cardiovascular modifiable risk factors is important foryounger populations.

\section{FUNDING:}

This work was supported by the Science and Technology Department of Sichuan, China (Grant No. 2020YJ0483, 2021 YJ0215).

\section{ACKNOWLEDGEMENT:}

The authors would like to thank the Science and Technology Department of Sichuan, China for their support. The authors also would like to express their gratitude to EditSprings (http://www.editsprings.com/) for the expert linguistic services provided.

\section{ETHICAL APPROVAL:}

The study was approved by the Medical Ethics Committee of the Third People's Hospital of Chengdu.

\section{CONFLICTOF INTEREST:}

The authors declared no conflict of interest.

\section{AUTHORS' CONTRIBUTION:}

XC: Conceptualisation, formal analysis, writing original draft. HL: Funding acquisition, supervision, writing review and editing. $X Y$ : Supervision; validation, writing review and editing.

SY, LQ: Data curation, investigation, writing review and editing. LC: Conceptualisation, Funding acquisition, supervision, writing review and editing.

\section{REFERENCES}

1. Wilmot KA, O'Flaherty M, Capewell S, Ford ES, Vaccarino V. Coronary heart disease mortality declines in the United States from 1979 through 2011: Evidence for stagnation in young adults, especially women. Circulation 2015; 132: 997-1002. doi: 10.1161/CIRCULATIONAHA.115. 015293.

2. Wang DZ, Shen CF, Zhang Y, Zhang H, Song GD, Li W, et al. Fifteen-year trend in incidence of acute myocardial infarction in Tianjin of China. Zhonghua Xin Xue Guan Bing Za Zhi 2017; 45:154-9. doi: 10.3760/cma.j. issn.02533758.2017.02.016. 
3. Arora S, Stouffer GA, Kucharska-Newton AM, Qamar A, Vaduganathan $M$, Pandey $A$, et al. Twenty year trends and sex differences in young adults hospitalised with acute myocardial infarction. Circulation 2019; 139: 1047-1056. doi: 10.1161/CIRCULATIONAHA.118.037137.

4. Piironen M, Ukkola O, Huikuri H, Havulinna AS, Koukkunen $\mathrm{H}$, Mustonen J, et al. Trends in long-term prognosis after acute coronary syndrome. Eur J Prev Cardiol 2017; 24:274-80. doi: 10.1177/2047487316679522.

5. Kannel WB, Dawber TR, Kagan A, Revotskie N, Stokes J 3rd. Factors of risk in the development of coronary heart disease--six year follow-up experience. The framingham study. Ann Intern Med 1961; 55:33-50. doi: 10. 7326/0003-4819-55-1-33.

6. Roth GA, Johnson C, Abajobir A, Abd-Allah F, Abera SF, Abyu $G$, et al. Global, regional, and national burden of cardiovascular diseases for 10 causes, 1990 to 2015. J Am Coll Cardiol 2017; 70:1-25. doi: 10.1016/j. jacc.2017. 04.052.

7. Chinese Society of Cardiology. CSC guidelines for the diagnosis and treatment of acute myocardial infarction in patients presenting with ST-segment elevation. Chin J Cardiol 2015; 43:380-93. doi: 10.3760/cma.j.issn. 0253-3758.2015.05.003.

8. Chinese society of cardiology. CSC guidelines for the diagnosis and treatment of acute coronary syndromes in patients presenting without persistent ST-segment elevation. Chin J Cardiol 2017; 45:359-376. doi: 10.3760/cma.j.issn.0253-3758.2017.05.003.

9. Carrillo X, Vilalta V, Cediel G, Fernandez-Nofrerias E, Rodriguez-Leor $\mathrm{O}$, Mauri J, et al. Trends in prevalence and outcomes of acute coronary syndrome associated with cocaine consumption: The RUTI-cocaine study. Int J Cardiol 2019; 283:23-7. doi: 10.1016/j.ijcard.2018. 12.026.

10. Sabbag A, Matetzky S, Porter A, lakobishvili Z, Moriel M, Zwas $D$, et al. Sex Differences in the management and 5year outcome of young patients ( $<55$ years) with acute coronary syndromes. Am J Med 2017; 130:1324.e151324.e22. doi: 10.1016/j.amjmed.2017.05.028.

11. Wang M, Luo X, Xu S, Liu W, Ding F, Zhang X, et al. Trends in smoking prevalence and implication for chronic diseases in China: Serial national cross-sectional surveys from 2003 to 2013. Lancet Respir Med 2019; 7:35-45. doi: 10. 1016/S2213-2600(18)30432-6.

12. Hu G, Zhou M, Liu J, Smith SC Jr, Ma C, Ge J, et al. Smoking and provision of smoking cessation interventions among inpatients with acute coronary syndrome in China: Findings from the improving care for cardiovascular disease in China-acute coronary syndrome project. Glob Heart 2020; 15:72. doi: 10. 5334/gh.784.

13. Freedman DS, Otvos JD, Jeyarajah EJ, Shalaurova I, Cupples LA, Parise $H$, et al. Sex and age differences in lipoprotein subclasses measured by nuclear magnetic resonance spectroscopy: The framingham study. Clin Chem 2004; 50:1189-1200. doi: 10.1373/clinchem. 2004.032763.

14. Wang X, Magkos F, Mittendorfer B.Sex differences in lipid and lipoprotein metabolism: It's not just about sex hormones. J Clin Endocrinol Metab 2011; 96:885-93. doi: 10.1210/jc.2010-2061.

15. Jortveit J, Pripp AH, Langørgen J, Halvorsen S. Incidence, risk factors and outcome of young patients with myocardial infarction. Heart 2020; 106:1420-6. doi: 10.1136/ heartjnl-2019-316067.

16. Li SY, Zhou MG, Ye T, Cheng LC, Zhu F, Cui CY, et al. Frequency of ST-segment elevation myocardial infarction, non-ST-segment myocardial infarction, and unstable angina: results from a Southwest Chinese Registry. Rev Cardiovasc Med 2021; 22:239-45. doi: 10.31083/j.rcm. 2021.01.103.

17. Yandrapalli S, Nabors C, Goyal A, Aronow WS, Frishman WH. Modifiable risk factors in young adults with first myocardial infarction. J Am Coll Cardiol 2019; 73:573-84. doi: 10.1016/j.jacc.2018.10.084.

18. Kayani WT, Ballantyne CM. Improving outcomes after myocardial infarction in the US population. J Am Heart Assoc 2018; 7:e008407. doi: 10.1161/JAHA.117.00 8407.

19. Jernberg $T$, Hasvold $P$, Henriksson $M$, Hjelm $H$, Thuresson M, Janzon M. Cardiovascular risk in post-myocardial infarction patients: Nationwide real world data demonstrate the importance of a long-term perspective. Eur Heart J 2015; 36:1163-70. doi: 10.1093/ eurheartj/ehu505.

20. Critchley JA, Capewell S. Mortality risk reduction associated with smoking cessation in patients with coronary heart disease: A systematic review. JAMA 2003; 290:86-97. doi: 10.1001/jama.290.1.86.

21. De Smedt D, De Bacquer D, De Sutter J, Dallongeville J, Gevaert S, De Backer G, et al. The gender gap in risk factor control: Effects of age and education on the control of cardiovascular risk factors in male and female coronary patients. The Euroaspire IV study by the European society of cardiology. Int J Cardiol 2016; 209:284-90. doi: 10.1016/j.ijcard.2016.02.015.

22. Desai NR, Udell JA, Wang Y, Spatz ES, Dharmarajan K, Ahmad T, et al. Trends in performance and opportunities for improvement on a composite measure of acute myocardial infarction care. Circ Cardiovasc Qual Outcomes 2019; 12:e004983. doi: 10.1161/CIRCOUTCOMES. 118.004983.

23. Xu JJ, Jiang L, Song Y, Yao Y, Jia SD, Liu Y, et al. Related factors and the long-term outcome after percutaneous coronary intervention of premature acute myocardial infarction. Zhonghua Xin Xue Guan Bing Za Zhi 2020; 48:655-660. doi: 10.3760/cma.j.cn112148-2019120800738. 